\title{
Evaluation with Heart Rate Variability for the Treatment Effect of Aminophylline in Patients with Bradycardia after Cervical Spinal Cord Injury: A Preliminary Study
}

\author{
Noriyuki Ishikawa1 ${ }^{1}$, Naohisa Miyakoshi ${ }^{2 *}$ Tetsuya Suzuki ${ }^{1}$, Akiko Misawa ${ }^{3}$, Yuichi Takano ${ }^{4}$, \\ Yoichi Shimada ${ }^{2}$ \\ ${ }^{1}$ Department of Orthopedic Surgery, Akita Red Cross Hospital, Akita, Japan; ${ }^{2}$ Department of Orthopedic Surgery, Akita University \\ Graduate School of Medicine, Akita, Japan; ${ }^{3}$ Department of Orthopedic Surgery, Akita Prefectural Center on Development and Disa- \\ bility, Akita, Japan; ${ }^{4}$ Iwai Orthopaedic Medical Hospital, Tokyo, Japan. \\ Email: *miyakosh@doc.med.akita-u.ac.jp
}

Received November $20^{\text {th }}, 2012$; revised February $25^{\text {th }}, 2013$; accepted March $18^{\text {th }}, 2013$

\begin{abstract}
After cervical spinal cord injury (SCI), the autonomic nervous system (ANS) becomes impaired and then, bradycardia can develop. In view of this, we performed to prescribe aminophylline as pharmacotherapy for bradycardia. The study population consisted of 36 patients with cervical SCI. Bradycardia developed in 20 patients (55.6\%), of these patients, 8 showed spontaneous recovery. Twelve patients had persistent bradycardia, therefore, aminophylline was administered at $0.5 \mathrm{mg} / \mathrm{kg} / \mathrm{hr}$ by intravenous infusion. Their average heart rate increased within 24 hours after the start of infusion. In heart rate variability analysis for 7 preliminarily selected patients, the spectral waveforms of "oligowave type" indicating ANS impairment tended to appear in relatively early phase after injury (i.e., 2 days to 2 weeks after injury), whereas "normal type" was observed in the late phase (i.e., at 4 weeks). "Sympathetic block type" was observed throughout the follow-up period (2 days to 4 weeks). "Sympathetic block type" was also observed in a non-bradycardic patient on day 2. These results underscore the importance of treating ANS impairment with aminophylline while keeping in mind that bradycardia can occur even in post-SCI patients without clinical manifestations.
\end{abstract}

Keywords: Spinal Cord Injury; Aminophylline; Heart Rate Variability; Autonomic Nervous System; Bradycardia

\section{Introduction}

After cervical spinal cord injury (SCI), sympathetic elements of the autonomic nervous system (ANS) become impaired and the parasympathetic nervous system eventually predominates, leading to ANS imbalance. As a result, bradycardia develops and the patient's general condition becomes unstable with emergence of hypotension, tracheostenosis, and increased airway secretions. In such patients, bradycardia may lead to cardiac arrest and should be actively treated. Pharmacotherapy usually consists of atropine sulfate, of which the effect is short-lived or may even be absent. Placement of a pacemaker, which is unavoidably invasive, is indicated in such cases. In view of this, we make it a rule to prescribe aminophylline as pharmacotherapy for bradycardia and the efficacy of this approach has been demonstrated [1].

Recently, there have been reports on assessment of ANS function by means of measuring heart rate variability [2]. According to this method, electrocardiographic

"Corresponding author.
RR intervals are continuously measured with a Holter electrocardiographic device, and the power spectrum density (PSD) is determined with the mathematical calculation, where a low frequency (LF) component and a high frequency (HF) component are extracted. The former is interpreted mainly as a representation of sympathetic tone, and the latter as that of the parasympathetic tone [3-7]. It is considered to be feasible to assess postSCI ANS function by this method.

The objectives of the present study were to examine the therapeutic effect of aminophylline on bradycardia and to preliminarily assess ANS function with heart rate variability analysis.

\section{Subjects and Methods}

The study population consisted of 36 patients with cervical SCI who had been emergently transported and then admitted to the Critical Care and Emergency Center of the first author's hospital within 24 hours after incurring the injury between 2008 and 2011. They comprised 27 
men and 9 women with an average age of 62.0 years. The American Spinal Injury Association (ASIA) Impairment Scale at the first presentation was: A in 7 patients, $B$ in $1, C$ in 11 , and $D$ in 17 . None of the study subjects had complicated injuries with Abbreviated Injury Scale (AIS) score 3 or greater.

Bradycardia was defined as heart rates $\leq 54$ beats per minute (bpm). Aminophylline was administered at 0.5 $\mathrm{mg} / \mathrm{kg} / \mathrm{hr}$ by intravenous infusion when heart rates $\leq 49$ bpm persisted. The aminophylline infusion was continued for a week as standard therapy, followed by oral theophylline medication at $400 \mathrm{mg} /$ day over the ensuing 4 weeks. Durations of this medication regimen were adjusted according to each patient's recovery state. Plasma drug levels were determined during the medication period. None of the patients included in this study had heart disease before injury.

Preliminary assessment of ANS function by heart rate variability analysis was performed on 9 occasions in 7 patients: 3 patients on day 2 post-injury and the other 4 patients at 2 weeks post-injury, and 2 of 3 patients assessed on day 2 post-injury underwent the analysis again at 4 weeks post-injury. Electrocardiograms of these patients were recorded with a Holter electrocardiographic device, and time-series data of RR intervals were extracted with a PC system (Holter Analyzer SCM-5000 System, Fukuda-Denshi, Tokyo, Japan). For these recording, the power spectrum density (PSD) is determined with the maximum entropy method (MEM), where a LF component $(0.04-0.15 \mathrm{~Hz})$ and a HF component $(0.15$ $0.4 \mathrm{~Hz}$ ) are extracted [2]. Analyzed spectral waveforms were classified into 3 types, i.e., "oligowave type" in which the LF component was extremely low with an unidentifiable HF component, "sympathetic block type" characterized by a low LF component and a predominant HF component, and "normal type". Patients presenting with atrial fibrillation were excluded from the analysis.

Because use of aminophylline was a conventional therapy for bradycardia, an approval from institutional review board was not required for this study. Informed consent for the assessment of ANS function by heart rate variability analysis was obtained from each patient. Paired t-test was used for comparison between the variables in different time points. p values less than 0.05 were considered statistically significant.

\section{Results}

The mean heart rate at the first presentation (on admission) was $76.3 \mathrm{bpm}$. Bradycardia developed in 20 patients (55.6\%) on the 3.8th hospital day post-injury on average. Of these patients, 8 showed spontaneous recovery while the remaining 12 had persistent bradycardia requiring aminophylline infusion. For the 8 patients showing spontaneous recovery, the mean heart rate at the first presentation was $78.5 \mathrm{bpm}$, and bradycardia had progressed to a mean heart rate of $50.3 \mathrm{bpm}$ by the 3.5th hospital day post-injury ( $<<0.05$, vs. baseline). Their heart rates were significantly recovered in 1 - 2 days (mean, 1.3 days) to $66.3 \mathrm{bpm}$ on average without treatment ( $\mathrm{p}<0.05$, vs. bradycardia state). In the 12 patients requiring aminophylline infusion, the mean heart rate was $70.9 \mathrm{bpm}$ at the first presentation and then had significantly dropped to $47.1 \mathrm{bpm}$ by the 3.9th hospital day post-injury ( $<0.05$, vs. baseline). Their average heart rate significantly increased to $59.3 \mathrm{bpm}$ within 24 hours after the start of aminophylline infusion ( $<<0.05$, vs. bradycardia state) (Figure 1).

In one patient, who required endotracheal intubation and respiration control due to respiratory paralysis at the first presentation, dopamine was administered for hypotension. None of the other patients had hypotension, regardless of whether or not there was bradycardia. None had signs of aminophylline intoxication or showed abnormal blood pressure elevation during the medication period. All patients were weaned uneventfully from the drugs and none required placement of a pacemaker.

Heart rate variability analysis was performed on 6 patients treated with aminophylline and 1 non-bradycardic patient. On day 2, "oligowave type" and "sympathetic block type" were seen in 1 and 2 patients, respectively (Table 1). At week 2 post-injury, "oligowave type” and "sympathetic block type" were seen in 1 and 3 patients, respectively. At week 4 post-injury, "sympathetic block type" and "normal type" were observed in one patient each.

In most of the cases displaying "sympathetic block type" waveforms, there was disappearance of diurnal variations which would usually be active at night in terms of the HF component, and the variations became predominant both in the daytime and at night. In the two cases treated with aminophylline and measured repeatedly, one case showed "oligowave type" on day 2 and

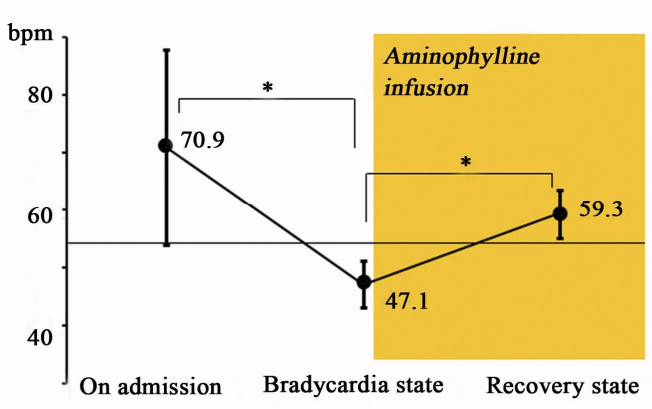

Figure 1. Changes of the heart rates before and after aminophylline infusion. Values are expressed as mean \pm standard deviation. ${ }^{*} \mathrm{p}<0.05$ (paired t-test). 
"sympathetic block type" at week 4 post-injury (Figure 2), and the other case showed "sympathetic block type" on day 2 and "normal type" at week 4 post-injury (Figure 3). The non-bradycardic patient exhibited "sympathetic block type” waveforms on day 2 post-injury.

Table1. Time-dependent appearance of waveforms analyzed with heart rate variability in patients with cervical spinal cord injury.

\begin{tabular}{cccc}
\hline & On day 2 & At 2 weeks & At 4 weeks \\
\hline Oligowave type & 1 & 1 & \\
Sympathetic block type & $2^{*}$ & 3 & 1 \\
Normal type & & & 1 \\
\hline
\end{tabular}

Data represents number of patients at each time point. All patients, except one patient, received aminophylline treatment. *Including one patient with no bradycardia and aminophylline treatment.

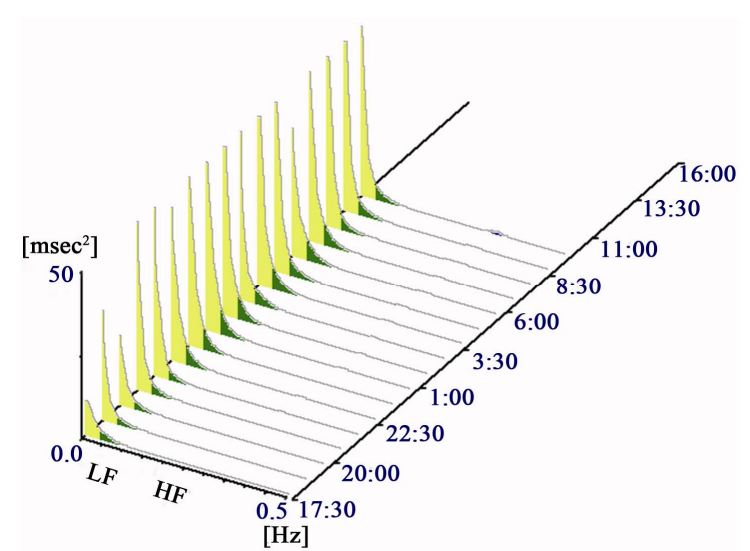

(a)

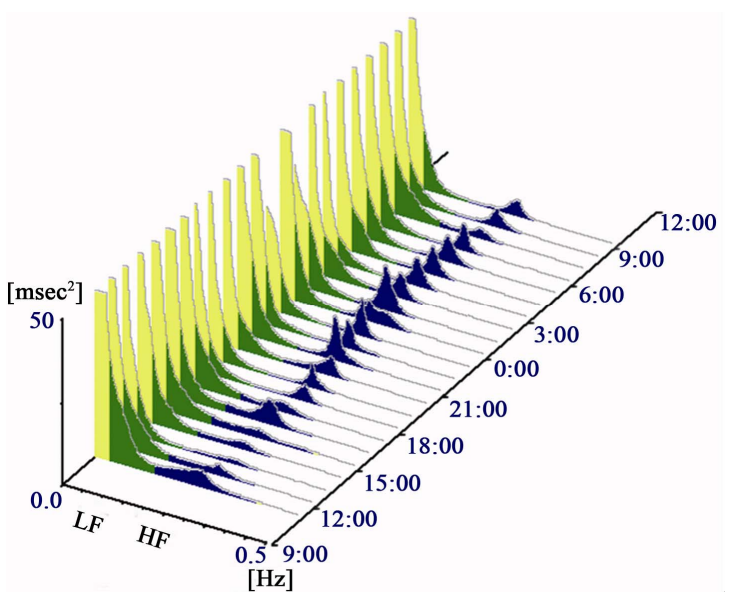

(b)

Figure 2. 62 y/o, male. ASIA Impairment Scale at the first presentation was A. Data from 2 days after injury showed typical "oligowave type" in which the LF component was extremely low with an unidentifiable HF component (a). Data from 4 weeks after injury showed "sympathetic block type" characterized by a low LF component and a predominant HF component (b).

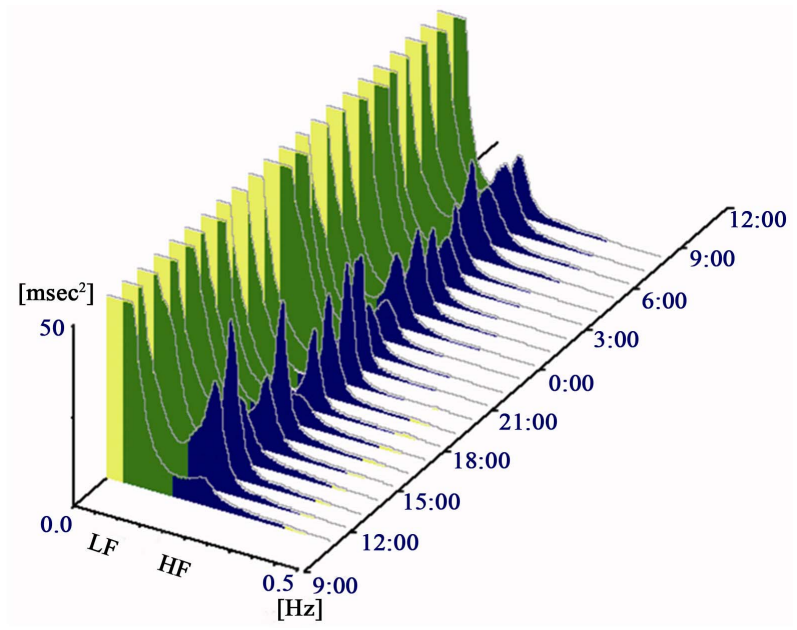

(a)

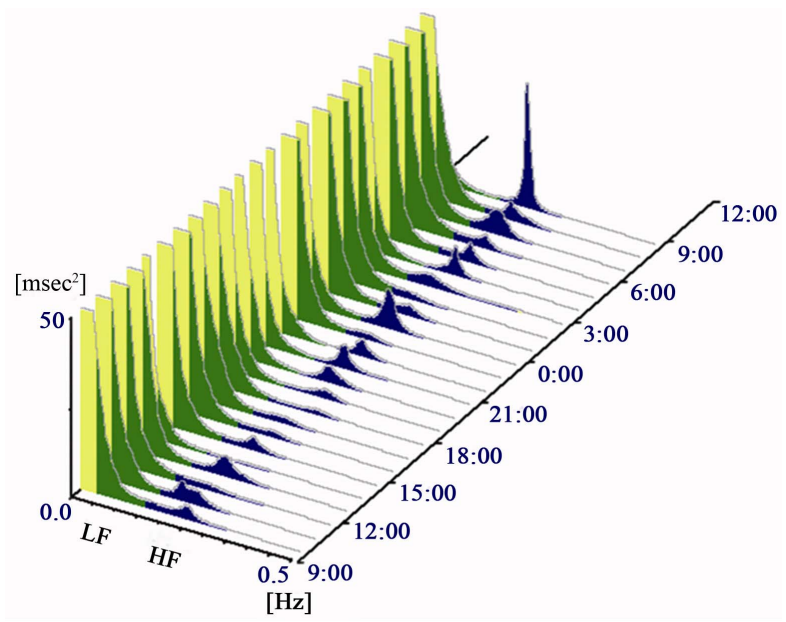

(b)

Figure 3. $63 \mathrm{y} / \mathrm{o}$, male. ASIA Impairment Scale at the first presentation was A. Data from 2 days after injury showed "sympathetic block type" characterized by the power spectrum of LF component does not reach 3 times of HF component and no circadian rhythm on the HF component (a). Data from 4 weeks after injury showed "normal type" with improvement of circadian rhythm (b).

\section{Discussion}

Bradycardia $\leq 50 \mathrm{bpm}$ necessitates treatment as this represents sick sinus syndrome. Therefore, patients persistently showing heart rates $\leq 49 \mathrm{bpm}$ are managed with aminophylline infusion while those showing heart rates $\leq$ 54 bpm are followed-up without intervention. Aminophylline is known to cause tachycardia, but allowed satisfactory control of bradycardia in the present series as well as in the previous case report [8]. No patient developed symptoms of toxicity and plasma drug concentrations remained within normal limits during the study treatment period. As a bronchodilating effect can also be expected from aminophylline, this drug is considered to 
be effective in patients with cervical SCI and respiratory palsy.

It has been reported that the LF component reflects sympathetic action and the HF component reflects the function of the parasympathetic action [3-7]. Generally, normal waveforms have 3 times more LF components than HF components. In this preliminary study, "oligowave type" showed ANS imbalance in which the LF component was extremely low with an unidentifiable HF component. Also, "sympathetic block type" indicated an impairment of sympathetic control characterized by a low LF component and a predominant HF component. The fact that two waveforms were noted on day 2 post-injury and at week 2 post-injury raises the possibility that the analysis detected ANS abnormalities in the acute phase after cervical SCI. The waveforms of "sympathetic block type" and "normal type" observed at week 4 post-injury might be a result being administered aminophylline. Repeated heart rate variability analysis thus may allow the process of ANS improvement during the course of treatment or spontaneous progression.

The present study also demonstrated ANS impairment that occurs in non-bradycardic patients as well as those with bradycardia. These results underscore the importance of treating ANS impairment while keeping in mind that bradycardia can occur even in post-SCI patients without clinical manifestations of this arrhythmia. This preliminary study gives physicians a new medicine choice when facing bradycardia caused by ANS impairment. However, the using dose of aminophylline should be paid more attention because the appropriate range may be narrow.

\section{Conclusion}

Bradycardia developed in $55.6 \%$ of patients just after cervical SCI. As pharmacotherapy for bradycardia, aminophylline was considered to be effective and safety in these patients. Preliminary assessment of ANS function by heart rate variability analysis showed that sympathetic action was suppressed and parasympathetic tone became hyperactive after cervical SCI. Sympathetic control inhibition may occur in non-bradycardic patients as well as those with bradycardia.

\section{Acknowledgements}

We wish to thank Dr. Kazuki Miura, Honorary Director of Sotoasahikawa Hospital, for data analysis of heart rate variability and for valuable discussion during the study.

\section{REFERENCES}

[1] N. Ishikawa and T. Takano, "Aminophylline for the Treatment of Bradycardia after Cervical Spinal Cord Injury," Journal of Spine Research, Vol. 1, No. 3, 2010, p.598.

[2] S. Fukui, K. Miura, H. Kurokawa, M. Sano, K. Sato, M. Sasaki and H. Ito, "Evalution of the Autonomic Nervous Function in Patients with Chronic Obstructive Pulmonary Disease Based on the Heart Rate Variability: Particularly Regarding the Presence or Absence of Parasympathetic Tone,” Akita Journal of Medicine, Vol. 34, No. 1, 2007, pp. 35-41.

[3] S. Akselrod, D. Gordon, F. A. Ubel, D. C. Shannon, A. C. Berger and R. J. Cohen, "Power Spectrum Analysis of Heart Rate Fluctuation: A Quantitative Probe of Beat-toBeat Cardiovascular Control,” Science, Vol. 213, No. 4504, 1981, pp. 220-222. doi:10.1126/science.6166045

[4] Task Force of the European Society of Cardiology and the North American Society of Pacing and Electrophysiology, "Heart Rate Variability: Standards of Measurement, Physiological Interpretation and Clinical Use," Circulation, Vol. 93, No. 5, 1996, pp. 1043-1065. doi:10.1161/01.CIR.93.5.1043

[5] R. D. Berger, J. P. Saul and R. J. Cohen, “Transfer Function Analysis of Autonomic Regulation. I. Canine Atrial Rate Response,” American Journal of Physiology, Vol. 256, No. 1, 1989, pp. H142-H152.

[6] V. K. Yeragani, K. Srinivasan, S. Vempati, R. Pohl and R. Balon, "Fractal Dimension of Heart Rate Time Series: An Effective Measure of Autonomic Function," Journal of Applied Physiology, Vol. 75, No. 6, 1993, pp. 2429-2438.

[7] B. Pomeranz, R. J. Macaulay, M. A. Caudill, I. Kutz, D. Adam, D. Gordon, K. M. Kilborn, A. C. Barger, D. C. Shannon, R. J. Cohen, et al., "Assessment of Autonomic Function in Humans by Heart Rate Spectral Analysis," American Journal of Physiology, Vol. 248, No. 1, 1985, pp. H151-H153.

[8] T. Sakamoto, T. Sadanaga and T. Okazaki, "Sequential Use of Aminophylline and Theophylline for the Treatment of Atropine-Resistant Bradycardia after Spinal Cord Injury: A Case Report,” Journal of Cardiology, Vol. 49, No. 2, 2007, pp. 91-96. 